\title{
NORI'S CONSTRUCTION AND THE SECOND ABEL-JACOBI MAP
}

\author{
KeNICHIRO KIMURA
}

\section{Introduction}

Let $k$ be a subfield of $\mathbb{C}$. Nori constructs an abelian category of mixed motives over $k$. One of the fundamental facts in his construction is the following $([7])$ :

Theorem 1.1 (Basic Lemma). Let $X$ be an affine scheme of finite type over $k$. Let $n$ be the dimension of $X$. Let $F$ be a weakly constructible sheaf on $X(\mathbb{C})$ for the usual topology. Then there is a Zariski open $U$ in $X$ with the properties below, where $j: U \rightarrow X$ denotes the inclusion.

(1) $\operatorname{dim} Y<n$ where $Y=X-U$.

(2) $H^{q}\left(X(\mathbb{C}), j ! j^{*} F\right)=0$ for $q \neq n$.

Here a sheaf $F$ on $X$ is weakly constructible if $X$ is the disjoint union of finite collection of locally closed subschemes $Y_{i}$ defined over $k$ such that the restrictions $\left.F\right|_{Y_{i}}$ are locally constant. Beilinson ([1], Lemma 3.3) proves this fact in all characteristics of the base field. Based on Theorem 1.1 Nori shows that affine $k$-varieties have a kind of "cellular decomposition". In section 2 of this note we give an exposition of the outcome if we apply this construction to etale cohomology. It can also be viewed as a partial exposition of $\ell$-adic realization of Nori's category of motives. The main result (Theorem 2.2) says that $R f_{*} \mathbb{Z}_{l}(a)$ for a variety $X \stackrel{f}{\rightarrow}$ Speck is a complex each component of which comes from a mixed motive. This gives an answer to a question asked by Jannsen in a remark in [4]. In [1] a similar result for perverse sheaves is proven. For the proof of Theorem 2.2 we first prove Proposition 2.1 which is a special case where $X$ is an affine variety. In the proof of this proposition we need to consider mapping cones of morphisms in a derived category. However mapping cone is not a well-defined construction in a derived category and if one does this in a naive way it causes problems with functoriality. The author is indebted to Uwe Jannsen for pointing out this problem and for telling the author how to fix it. In fact the proof of Proposition 2.1 in this paper is due to Jannsen. We learned about Nori's category in [5].

In section 3, we give a simple description of the second $\ell$-adic Abel-Jacobi map for certain algebraic cycles on a smooth projective variety. We briefly recall the definition of the $\ell$-adic Abel-Jacobi map. Let $X \stackrel{f}{\rightarrow}$ Speck be a smooth projective variety of dimension $n$. We denote the absolute Galois group of $k$ by $G_{k}$. For an algebraic cycle $z$ on $X$ of codimension $i$ the class

Received by the editors October 17, 2006. 


$$
[z] \in H_{\text {cont }}^{2 i}\left(X, \mathbb{Z}_{l}(i)\right)
$$

is defined. Here $H_{\text {cont }}^{2 i}\left(X, \mathbb{Z}_{l}(i)\right)$ is continuous etale cohomology. The usual cycle class $c l(z)=c l^{0}(z)$ is the image of $[z]$ under the edge homomorphism

$$
H_{\text {cont }}^{2 i}\left(X, \mathbb{Z}_{l}(i)\right) \rightarrow H^{2 i}\left(\bar{X}, \mathbb{Z}_{l}(i)\right) .
$$

The Hochschild-Serre spectral sequence

$$
E_{2}^{p, q}=H^{p}\left(G_{k}, H^{q}\left(\bar{X}, \mathbb{Z}_{l}(i)\right)\right) \Longrightarrow H_{\text {cont }}^{2 i}\left(X, \mathbb{Z}_{l}(i)\right)
$$

induces higher classes

$$
c l^{j}: \operatorname{Ker} c l^{j-1} \rightarrow H^{j}\left(G_{k}, H^{2 i-j}\left(\bar{X}, \mathbb{Q}_{l}(i)\right)\right) .
$$

We refer the reader to [4] for more details. See also [8].

Let $z \in C H^{i}(X)$ be an algebraic cycle such that $0=\operatorname{cl}^{1}(z) \in H^{1}\left(G_{k}, H^{2 i-1}\left(\bar{X}, \mathbb{Z}_{l}(i)\right)\right)$. In Theorem 3.1 we give a simple description of the push-out of $c l^{2}(z) \in H^{2}\left(G_{k}, H^{2 i-2}\left(\bar{X}, \mathbb{Q}_{l}(i)\right)\right)$ by a quotient map $H^{2 i-2}\left(\bar{X}, \mathbb{Q}_{l}(i)\right)$ $\rightarrow H^{2 i-2}\left(\bar{X}, \mathbb{Q}_{l}(i)\right) / H_{H}^{2 i-2}\left(\bar{X}, \mathbb{Q}_{l}(i)\right)$ for a certain multiple hypersurface section $H$ of $X$.

G. Welters([9]) also gives a description of the second Abel-Jacobi map for zero-cycles. It would be interesting to compare these two descriptions.

\section{Nori's construction}

Let $k$ be a subfield of $\mathbb{C}$. In this note a variety is an integral separated scheme of finite type over $k$. All schemes and morphisms between them are defined over $k$. For a variety $X, \bar{X}$ denotes $X \times_{\operatorname{Spec} k} \operatorname{Spec} \bar{k}$ where $\bar{k}$ is an algebraic closure of $k$.

Theorem 2.1. Let $X$ be an affine scheme of finite type of dimension n. Let $F$ be a constructible sheaf on $X(\mathbb{C})$ for the usual topology. Then there is a Zariski open $U$ in $X$ with the properties below, where $j: U \rightarrow X$ denotes the inclusion.

(1) $\operatorname{dim} Y<n$ where $Y=X-U$.

(2) $H^{q}\left(X(\mathbb{C}), j ! j^{*} F\right)=0$ for $q \neq n$.

Let $X^{\prime}$ be the largest open subscheme of $X$ such that $X^{\prime}$ is smooth and $\left.F\right|_{X^{\prime}}$ is locally constant. As in Remark 1.1 in [7] the open set $U$ depends only on the open $X^{\prime}$ and not on $F$.

Fix an integer $a$ and a prime $l$. We are going to use Theorem 2.1 in the case where $F$ is an etale sheaf of the form $j_{V !} j_{V}^{*} \mathbb{Z} / l^{m} \mathbb{Z}(a)$ for an open subscheme $V \stackrel{j_{V}}{\hookrightarrow} X$. In this case $H^{q}\left(X(\mathbb{C}), j_{!} j^{*} F\right)$ in the assertion 2 of Theorem 2.1 is isomorphic to $H^{q}\left(\bar{X}_{e t}, j_{!} j^{*} F\right)$. The latter group is also denoted $H^{q}(\bar{X}, \bar{Y}, F)$, where $Y \subset X$ is a closed complement of $V$ in $X$.

Let $X$ be an affine variety and let $Z$ be a proper closed subscheme of $X$. Let $j$ be the inclusion $X-Z \rightarrow X$. By applying Theorem 2.1 to $F=j ! j^{*} \mathbb{Z} / l^{m} \mathbb{Z}(a)$ we obtain the following (cf. [7] Remark 1.2): 
Corollary 2.1. Let $X$ be an affine variety of dimension $n$ and let $Z$ be a closed subscheme of dimension $<n$. Then there exists a closed subscheme $Y$ of $X$ of dimension $<$ $n$ which contains $Z$ such that

$$
H^{q}\left(\bar{X}, \bar{Y}, \mathbb{Z} / l^{m} \mathbb{Z}(a)\right)=0 \text { for } \quad q \neq n
$$

for all $m \geq 1$.

By Corollary 2.1 there is a filtration by closed subschemes

$$
\emptyset=X_{-1} \subset X_{0} \subset \cdots \subset X_{n}=X
$$

such that $H^{q}\left(\bar{X}_{i}, \bar{X}_{i-1}, \mathbb{Z} / l^{m} \mathbb{Z}(a)\right)=0$ for $q \neq i$ and for $m \geq 1$.

Let $f: X \rightarrow$ Spec $k$ be the structure morphism. We consider $R f_{*} \mathbb{Z}_{l}(a)$ in $D^{b}\left(\operatorname{Sh}\left(\operatorname{Spec} k_{e t}\right)^{\mathbb{Z}_{l}}\right)$. Here, for a variety $X, S h\left(X_{e t}\right)^{\mathbb{Z}_{l}}$ denotes the category of $\ell$-adic sheaves of Jannsen([2], (6.9)). $S h\left(X_{e t}\right)^{\mathbb{Z}_{l}}$ has enough injectives.

Proposition 2.1. Let $X$ be an affine variety of dimension $n$ and take a filtration

$$
\emptyset=X_{-1} \subset X_{0} \subset \cdots \subset X_{n}=X
$$

by closed subschemes as above.

(1) Let $D(X)$ be the complex

$$
\begin{aligned}
& 0 \rightarrow H^{0}\left(\bar{X}_{0}, \mathbb{Z}_{l}(a)\right) \rightarrow \cdots \rightarrow H^{i}\left(\bar{X}_{i}, \bar{X}_{i-1}, \mathbb{Z}_{l}(a)\right) \rightarrow \cdots \rightarrow \\
& H^{n}\left(\bar{X}, \bar{X}_{n-1}, \mathbb{Z}_{l}(a)\right) \rightarrow 0
\end{aligned}
$$

where the maps between components are the boundary map of cohomology. Let $f: X \rightarrow$ Speck be the structure morphism. Then there is a natural isomorphism

$$
\mathbb{R} f_{*} \mathbb{Z}_{l}(a) \simeq D(X)
$$

in $D^{b}\left(\operatorname{Sh}(\text { Speck })^{\mathbb{Z}_{l}}\right)$.

(2) Let $Y \stackrel{j_{Y}}{\hookrightarrow} X$ be an affine open subscheme of $X$ and

$$
\emptyset=Y_{-1} \subset Y_{0} \subset \cdots \subset Y_{n}=Y
$$

be a filtration by closed subschemes such that $H^{q}\left(\bar{Y}_{i}, \bar{Y}_{i-1}, \mathbb{Z} / l^{m} \mathbb{Z}(a)\right)=0$ for $q \neq i$ and for $m \geq 1$. Assume that $Y_{i} \subset X_{i}$ for each $i$. Let $D(Y)$ be the complex

$$
\begin{aligned}
0 \rightarrow H^{0}\left(\bar{Y}_{0}, \mathbb{Z}_{l}(a)\right) & \rightarrow \cdots \rightarrow H^{i}\left(\bar{Y}_{i}, \bar{Y}_{i-1}, \mathbb{Z}_{l}(a)\right) \rightarrow \cdots \\
& \rightarrow H^{n}\left(\bar{Y}, \bar{Y}_{n-1}, \mathbb{Z}_{l}(a)\right) \rightarrow 0 .
\end{aligned}
$$

Let $g=f \circ j_{Y}: Y \rightarrow$ Speck. Then the isomorphisms $\mathbb{R} f_{*} \mathbb{Z}_{l}(a) \simeq D(X)$ and $R g_{*} \mathbb{Z}_{l}(a) \simeq D(Y)$ are compatible with the pull-back $j_{Y}^{*}$.

The proof given here is due to Jannsen. 
Proof. 1. First we fix notation and the signs. The mapping cone of $f: A \rightarrow B$. between two cohomological complexes is given in degree $i$ by

$$
A^{i+1} \oplus B^{i}, \quad \text { with differential } \quad d(a, b)=(-d a, d b+f(a)) .
$$

For $0 \leq a \leq n$ let $X_{a}^{o}=X_{a}-X_{a-1}, j_{a}: X_{a}^{o} \hookrightarrow X_{a}, i_{a}: X_{a} \hookrightarrow X$ and $\nu_{a}: X_{a} \hookrightarrow X_{a+1}$. We fix an injective resolution $I_{m}$ of $\mathbb{Z} / l^{m} \mathbb{Z}(a)$ in the big etale site over Speck. It gives a complex $B_{m}(Y)=g_{*}\left(\left.I_{m}^{*}\right|_{Y}\right)$ for each $g: Y \rightarrow$ Speck which represents $R g_{*} \mathbb{Z} / l^{m} \mathbb{Z}(a)$ and which is contravariantly functorial in $Y$.

Let $D_{m}(X)$ be the complex

$$
\begin{aligned}
0 \rightarrow H^{0}\left(\bar{X}_{0}, \mathbb{Z} / l^{m} \mathbb{Z}(a)\right) \rightarrow \cdots & \rightarrow H^{i}\left(\bar{X}_{i}, \bar{X}_{i-1}, \mathbb{Z} / l^{m} \mathbb{Z}(a)\right) \rightarrow \\
& \cdots \rightarrow H^{n}\left(\bar{X}, \bar{X}_{n-1}, \mathbb{Z} / l^{m} \mathbb{Z}(a)\right) \rightarrow 0 .
\end{aligned}
$$

We construct a chain of quasi-isomorphisms between $D_{m}(X)$ and $B_{m}(X)$.

We have a sequence of morphisms of complexes

$$
B_{m}\left(X_{n}\right) \stackrel{\nu_{n-1}^{*}}{\rightarrow} B_{m}\left(X_{n-1}\right) \stackrel{\nu_{n-2}^{*}}{\longrightarrow} \cdots \stackrel{\nu_{1}^{*}}{\rightarrow} B_{m}\left(X_{1}\right) \stackrel{\nu_{0}^{*}}{\rightarrow} B_{m}\left(X_{0}\right) .
$$

Let $C_{i}$ be the complex Cone $\left(B_{m}\left(X_{i}\right) \rightarrow B_{m}\left(X_{i-1}\right)\right)[-1]$.

For each $i$ there is a canonical quasi-isomorphism

$$
j_{i !}\left(\mathbb{Z} /\left.l^{m} \mathbb{Z}(a)\right|_{X_{i}^{\circ}}\right) \stackrel{\sim}{\rightarrow} \text { Cone }\left(I_{m X_{i}}^{\cdot} \rightarrow \nu_{i-1_{*}} I_{m X_{i-1}}^{\cdot}\right)[-1] .
$$

because the morphism defining the cone canonically factorizes through $\left(\nu_{i-1}\right)_{*}\left(\nu_{i-1}\right)^{*} I_{m_{X_{i}}}$. The target of the above map is a complex of injective sheaves. Hence the complex $C_{i}$ represents $R f_{*} j_{i !}\left(\mathbb{Z} /\left.l^{m} \mathbb{Z}(a)\right|_{X_{i}^{\circ}}\right)$.

Consider the following triple complex:
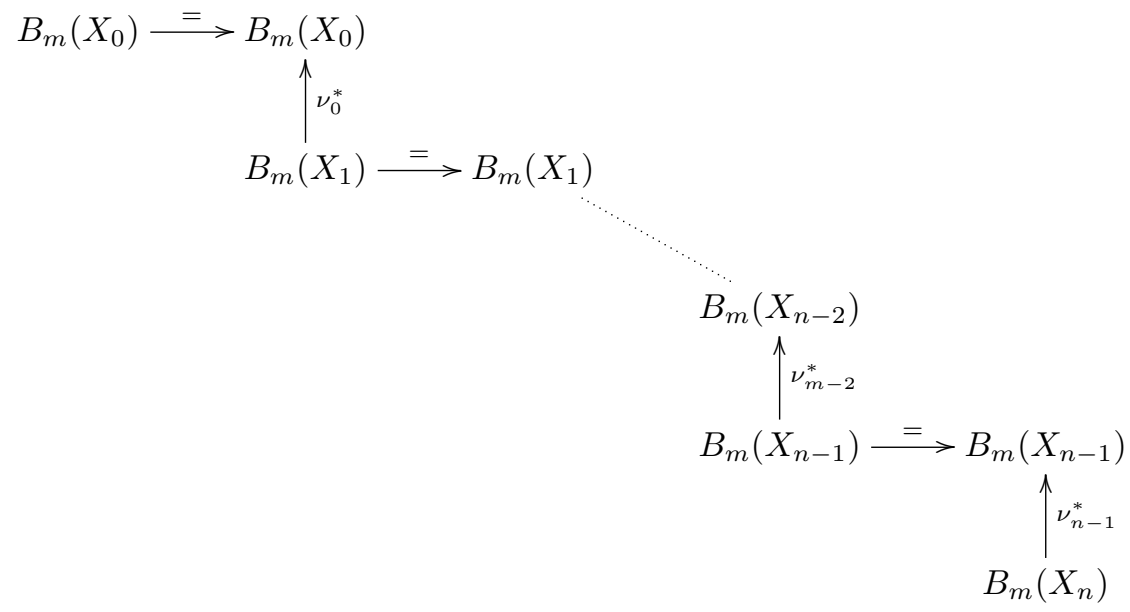

This induces a double complex $C$ :

$$
C_{0} \stackrel{\partial_{0}}{\rightarrow} C_{1}[1] \stackrel{\partial_{1}}{\rightarrow} C_{2}[2] \rightarrow \ldots \rightarrow C_{n-1}[n-1] \rightarrow C_{n}[n]
$$


(by taking the total complexes in the 'columns'). In explicit terms, $\partial_{i}$ is
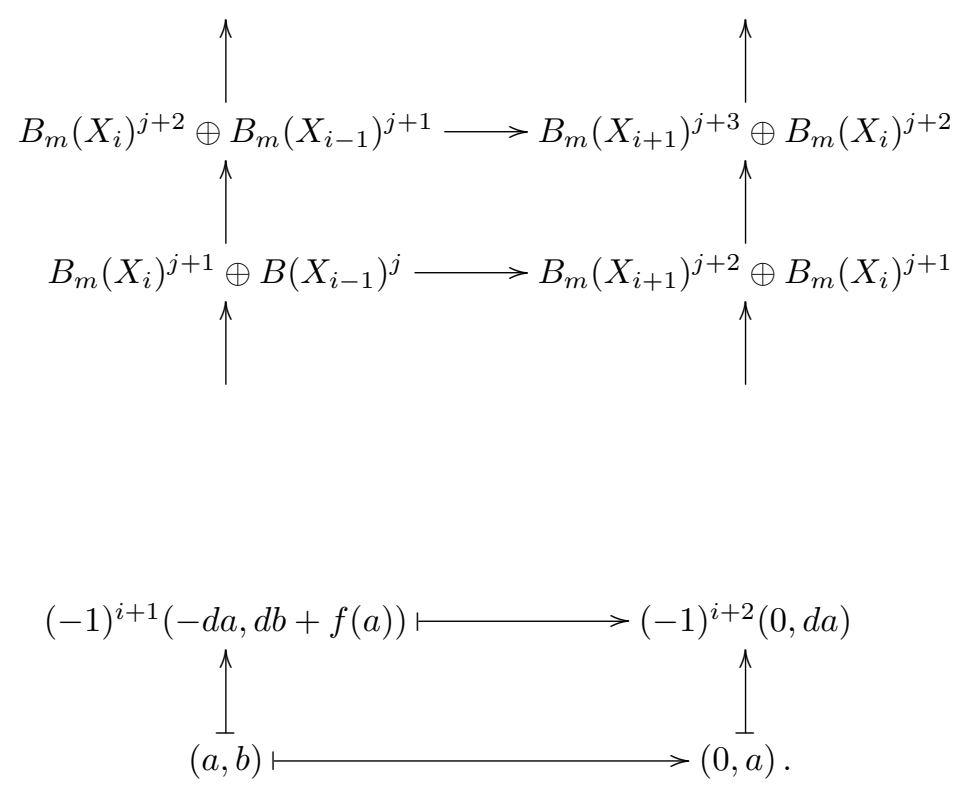

Now by assumption, we have

$$
H^{k}\left(C_{i}[i]\right)= \begin{cases}0 & , k \neq 0 \\ H^{i}\left(\bar{X}_{i}, \bar{X}_{i-1}, \mathbb{Z} / \ell^{m} \mathbb{Z}(a)\right) & , k=0 .\end{cases}
$$

Thus we get a canonical quasi-isomorphism of complexes

$$
\operatorname{Tot}(C) \stackrel{\sim}{\leftarrow} \operatorname{Tot}\left(t_{\leq 0} C\right) \stackrel{\sim}{\rightarrow} \operatorname{Tot}\left(t_{\leq 0} C / t_{\leq-1} C\right)=D_{m}(X)
$$

where $t_{\leq \nu} C$ means the canonical truncation of $C$ along columns, i.e., the complex

$$
\tau_{\leq \nu} C_{0} \rightarrow \tau_{\leq \nu}\left(C_{1}[1]\right) \rightarrow \ldots \rightarrow \tau_{\leq \nu}\left(C_{n}[n]\right) .
$$

The boundary map

$$
H^{i}\left(X_{i}, X_{i-1}\right) \rightarrow H^{i+1}\left(X_{i+1}, X_{i}\right)
$$

is the composition

$$
H^{i}\left(X_{i}, X_{i-1}\right) \rightarrow H^{i}\left(X_{i}\right) \rightarrow H^{i+1}\left(X_{i+1}, X_{i}\right)
$$

which is induced by

$$
\begin{aligned}
C_{i} & =C \text { Cone }\left(B\left(X_{i}\right) \rightarrow B\left(X_{i-1}\right)\right)[-1] \rightarrow B\left(X_{i}\right) \\
& \rightarrow \text { Cone }\left(B\left(X_{i+1}\right) \rightarrow B\left(X_{i}\right)\right)=C_{i+1}[1] .
\end{aligned}
$$

Explicitly

$$
\begin{aligned}
B\left(X_{i}\right)^{j} \oplus B\left(X_{i-1}\right)^{j-1} \rightarrow B\left(X_{i}\right)^{j} \rightarrow B\left(X_{i+1}\right)^{j+1} \oplus B\left(X_{i}\right)^{j} \\
(a, b) \mapsto a \mapsto(0, a) .
\end{aligned}
$$

Above $\operatorname{Tot}(C)$ is the total complex associated to the double complex, obtained by keeping the horizontal differentials and changing the differential in the $i$-th column by $(-1)^{i}$. With this normalization it is clear that via projection to $B_{m}\left(X_{n}\right)=B_{m}(X)$, 
$\operatorname{Tot}(C)$ is canonically quasi-isomorphic to $B_{m}\left(X_{n}\right)=B_{m}(X)$ : We have $k e r \partial_{i}=$ $i m \partial_{i-1}$ for all $i \leq n$, and coker $\partial_{n}=B_{m}\left(X_{n}\right)[n]$.

As in the proof of Proposition 1.1(a) of [2], one can construct an injecive resolution $\left(I_{m}\right)_{m}$ of the projective system $\left(\mathbb{Z} / l^{m} \mathbb{Z}(a)\right)_{m}$ on the big etale site.

2. All the maps in the construction of the isomorphism $\mathbb{R} f_{*} \mathbb{Z}_{l}(a) \simeq D(X)$ are compatible with the pull-back $j_{Y}^{*}$.

In general we use $\check{C}$ ech construction. Let $X$ be a variety of dimension $n$. Let $I$ be a finite set $\{1, \cdots, s\}$ and let $U_{i}(i \in I)$ be a covering of $X$ by affine open subschemes. For any subset $J \subset I$ we denote the intersection $\bigcap_{i \in J} U_{i}$ by $U_{J}$. We denote the open immersion $U_{J} \hookrightarrow X$ by $j_{J}$. For $U_{I}=\cap_{i \in I} U_{i}$ choose a filtration by closed subschemes $\emptyset=U_{I}^{-1} \subset U_{I}^{0} \subset U_{I}^{1} \subset \cdots U_{I}^{n-1} \subset U_{I}^{n}=U_{I}$ such that $H^{j}\left(\overline{U_{I}^{i}}, \overline{U_{I}^{i-1}}, \mathbb{Z} / l^{m} \mathbb{Z}(a)\right)=0$ for $i \neq j$ and for $m \geq 1$. When the filtrations $U_{J}^{j}$ of $U_{J}$ are defined for all subsets $J \subset I$ with $p+1$ elements and for $0 \leq j \leq n-1$, then, by Corollary 2.1, for subsets $J \subset I$ with $p$ elements the filtrations $\emptyset=U_{J}^{-1} \subset U_{J}^{0} \subset U_{J}^{1} \subset \cdots U_{J}^{n-1} \subset U_{J}^{n}=U_{J}$ can be chosen so that $H^{j}\left(\overline{U_{J}^{i}}, \overline{U_{J}^{i-1}}, \mathbb{Z} / l^{m} \mathbb{Z}(a)\right)=0$ for $i \neq j$ and for $m \geq 1$ and also that $U_{J^{\prime}}^{i} \subset U_{J}^{i}$ for any subset $J^{\prime} \subset I$ which strictly contains $J$ and for $0 \leq i \leq n$.

Let $I_{m}$ be the injective resolution of $\mathbb{Z} / l^{m} \mathbb{Z}(a)$ on the big etale site taken in the proof of Proposition 2.1. Recall the notation $B_{m}(Y):=g_{*}\left(\left.I_{m}^{\cdot}\right|_{Y}\right)$ for $g: Y \rightarrow$ Speck. We have the $\check{C}$ ech complex

$$
0 \rightarrow \underset{i \in I}{\oplus} B_{m}\left(U_{\{i\}}\right) \rightarrow \underset{i_{0}<i_{1}}{\oplus} B_{m}\left(U_{\left\{i_{0}, i_{1}\right\}}\right) \rightarrow \cdots \rightarrow B_{m}\left(U_{I}\right) \rightarrow 0 .
$$

The total complex associated to this double complex is quasi-isomorphic to $B_{m}(X)$. By Proposition 2.1 we obtain the following.

Theorem 2.2. The complex $R f_{*} \mathbb{Z}_{l}(a)$ is isomorphic to the total complex associated to the double complex

$$
0 \rightarrow \underset{i \in I}{\oplus} D\left(U_{\{i\}}\right) \rightarrow \underset{i_{0}<i_{1}}{\oplus} D\left(U_{\left\{i_{0}, i_{1}\right\}}\right) \rightarrow \cdots \rightarrow D\left(U_{\{1, \cdots, s\}}\right) \rightarrow 0
$$

in $D^{b}\left(S h\left(\operatorname{Spec}_{e t}\right)^{\mathbb{Z}_{l}}\right)$.

\section{A simple description of the second $\ell$-adic Abel-Jacobi map}

Let $X$ be a smooth projective variety of dimension $n$. Let $z \in C H^{i}(X)$ be an algebraic cycle which is homologous to 0 . Let $c l^{1}(z)$ be the image of $z$ under the $\ell$-adic Abel-Jacobi map

$$
c l^{1}: C H^{i}(X)_{\mathrm{hom}} \rightarrow H^{1}\left(G_{k}, H^{2 i-1}\left(\bar{X}, \mathbb{Z}_{l}(i)\right)\right) .
$$

Assume further that the cycle $z$ satisfies the following condition:

Let $q=2 i-1-n$. Then there exists a smooth multiple hypersurface section $H$ of $X$ of codimension $q$ which supports the cycle $z$ such that $z$ is homologous to 0 on $H$. 
For example if $i=n$ then by Proposition 4.8 of [3] (see also Proposition 5.3 of [6]) such a $H$ always exists. Let $|z|$ be the support of $z$ and let $Y=X-|z|$. Let $U=X-H$ and let $j: U \subset Y$ be the inclusion.

Let $g: Y \rightarrow$ Speck be the structure morphism. The 2-extension

$$
0 \rightarrow H^{2 i-2}\left(\bar{Y}, \mathbb{Q}_{l}(i)\right) \rightarrow \frac{R g_{*} \mathbb{Q}_{l}(i)^{2 i-2}}{\operatorname{Im} \partial^{2 i-3}} \stackrel{\partial^{2 i-2}}{\rightarrow} \operatorname{Ker}^{2 i-1} \rightarrow H^{2 i-1}\left(\bar{Y}, \mathbb{Q}_{l}(i)\right) \rightarrow 0
$$

is denoted as $\chi_{2 i-2}(Y)$ in [4].

Assume that $0=c l^{1}(z) \in H^{1}\left(G_{k}, H^{2 i-1}\left(\bar{X}, \mathbb{Z}_{l}(i)\right)\right)$. Then by Theorem 1 in [4] the class $-c l^{2}(z) \in \operatorname{Ext}_{G_{k}}^{2}\left(\mathbb{Q}_{l}, H^{2 i-2}\left(\bar{Y}, \mathbb{Q}_{l}(i)\right)\right)$ is the pull-back of $\chi_{2 i-2}(Y)$ by the splitting $c l^{1}(z): \mathbb{Q}_{l} \rightarrow H^{2 i-1}\left(\bar{Y}, \mathbb{Q}_{l}(i)\right)$.

Let $\mathcal{C}$ be the complex

$$
0 \rightarrow H^{2 i-1}\left(\bar{X}, \mathbb{Q}_{l}(i)\right) \rightarrow H^{2 i-1}\left(\bar{Y}, \mathbb{Q}_{l}(i)\right) \rightarrow H_{|z|}^{2 i}\left(\bar{X}, \mathbb{Q}_{l}(i)\right) \rightarrow 0
$$

and let $\mathcal{C}_{H}$ be the complex

$$
\begin{gathered}
0 \rightarrow H^{2(i-q)-1}\left(\bar{H}, \mathbb{Q}_{l}(i-q)\right) \rightarrow H^{2(i-q)-1}\left(\overline{H \cap Y}, \mathbb{Q}_{l}(i-q)\right) \\
\rightarrow H_{|z|}^{2(i-q)}\left(\bar{H}, \mathbb{Q}_{l}(i-q)\right) \rightarrow 0 .
\end{gathered}
$$

There is the Gysin map $i_{H *}: \mathcal{C}_{H} \rightarrow \mathcal{C}$. From the definition of $q$ the map $i_{H *}$ : $H^{2(i-q)-1}\left(\bar{H}, \mathbb{Q}_{l}(i-q)\right) \rightarrow H^{2 i-1}\left(\bar{X}, \mathbb{Q}_{l}(i)\right)$ is surjective by hard Lefschetz theorem. So the cycle class $c l^{1}(z) \in \operatorname{Ext}_{G_{k}}^{1}\left(\mathbb{Q}_{l}, H^{2 i-1}\left(\bar{X}, \mathbb{Q}_{l}(i)\right)\right)$ is the image of $c l^{1}(z) \in$ $\operatorname{Ext}_{G_{k}}^{1}\left(\mathbb{Q}_{l}, H^{2(i-q)-1}\left(\bar{H}, \mathbb{Q}_{l}(i-q)\right)\right)$ under the Gysin map. It is also equal to the pushout by the quotient $H^{2(i-q)-1}\left(\bar{H}, \mathbb{Q}_{l}(i-q)\right) \rightarrow \frac{H^{2(i-q)-1}\left(\bar{H}, \mathbb{Q}_{l}(i-q)\right)}{\partial H^{2 i-2}\left(\bar{U}, \mathbb{Q}_{l}(i)\right)}$. By our condition on $H$, there is a splitting

$$
c l^{1}(z): \mathbb{Q}_{l} \rightarrow \frac{H^{2(i-q)-1}\left(\overline{H \cap Y}, \mathbb{Q}_{l}(i-q)\right)}{\partial H^{2 i-2}\left(\bar{U}, \mathbb{Q}_{l}(i)\right)} .
$$

Theorem 3.1. The push-out of $-c l^{2}(z) \in \operatorname{Ext}_{G_{k}}^{2}\left(\mathbb{Q}_{l}, H^{2 i-2}\left(\bar{Y}, \mathbb{Q}_{l}(i)\right)\right)$ by the quotient $H^{2 i-2}\left(\bar{Y}, \mathbb{Q}_{l}(i)\right) \rightarrow \frac{H^{2 i-2}\left(\bar{Y}, \mathbb{Q}_{l}(i)\right)}{H_{Y-U}^{2 i-2}\left(\bar{Y}, \mathbb{Q}_{l}(i)\right)}$ is given by the pull-back of the 2-extension

$$
\begin{aligned}
0 & \rightarrow \frac{H^{2 i-2}\left(\bar{Y}, \mathbb{Q}_{l}(i)\right)}{H_{Y-U}^{2 i-2}\left(\bar{Y}, \mathbb{Q}_{l}(i)\right)} \rightarrow H^{2 i-2}\left(\bar{U}, \mathbb{Q}_{l}(i)\right) \rightarrow H_{Y-U}^{2 i-1}\left(\bar{Y}, \mathbb{Q}_{l}(i)\right) \rightarrow \\
& \frac{H_{Y-U}^{2 i-1}\left(\bar{Y}, \mathbb{Q}_{l}(i)\right)}{\partial H^{2 i-2}\left(\bar{U}, \mathbb{Q}_{l}(i)\right)} \rightarrow 0
\end{aligned}
$$

by $c^{1}(z): \mathbb{Q}_{l} \rightarrow \frac{H_{Y-U}^{2 i-1}\left(\bar{Y}, \mathbb{Q}_{l}(i)\right)}{\partial H^{2 i-2}\left(\bar{U}, \mathbb{Q}_{l}(i)\right)}$.

Remark. When $i$ is equal to $n, H_{Y-U}^{2 n-2}\left(\bar{Y}, \mathbb{Q}_{l}(n)\right)$ is generated by the cohomology class of $H(1)$. So we do not lose too much information by the push-out.

Proof. We have an exact triangle

$$
\left.R g_{*} R i_{H} ! \mathbb{Q}_{l}(i) \stackrel{i_{H}}{\rightarrow} R g_{*} \mathbb{Q}_{l}(i) \stackrel{j^{*}}{\rightarrow} R(g \circ j)_{*} \mathbb{Q}_{l}(i)\right|_{U} \rightarrow
$$


We denote this triangle by $A \stackrel{i_{H} *}{\rightarrow} B \stackrel{j^{*}}{\rightarrow} C \rightarrow$. The 2-extension $\chi_{2 i-2}(Y)$ is given by

$$
0 \rightarrow H^{2 i-2}(\bar{Y}) \rightarrow \frac{B^{2 i-2}}{\partial_{B}^{2 i-3}\left(B^{2 i-3}\right)} \stackrel{\partial_{B}^{2 i-2}}{\rightarrow} \operatorname{Ker}_{B}^{2 i-1} \rightarrow H^{2 i-1}(\bar{Y}) \rightarrow 0 .
$$

Let $C_{2}$ be the complex

$$
\begin{aligned}
0 \rightarrow H^{2 i-2}(\bar{Y}) & \rightarrow \frac{\left(j^{*}\right)^{-1} \operatorname{Ker} \partial_{C}^{2 i-2}+i_{H *}\left(A^{2 i-2}\right)}{\partial_{B}^{2 i-3}\left(B^{2 i-3}\right)} \stackrel{\partial_{B}^{2 i-2}}{\rightarrow} \\
i_{H *}\left(\operatorname{Ker}_{A}^{2 i-1}\right) & \rightarrow \frac{H_{Y-U}^{2 i-1}(\bar{Y})}{\partial\left(H^{2 i-2}(\bar{U})\right)} \rightarrow 0 .
\end{aligned}
$$

Let $C_{3}$ be the complex

$$
\begin{aligned}
& 0 \rightarrow \frac{H^{2 i-2}(\bar{Y})}{\operatorname{Im}\left(i_{H *}\left(\operatorname{Ker} \partial_{A}^{2 i-2}\right)\right)} \rightarrow \frac{\left(j^{*}\right)^{-1} \operatorname{Ker} \partial_{C}^{2 i-2}+i_{H *}\left(A^{2 i-2}\right)}{\partial_{B}^{2 i-3}\left(B^{2 i-3}\right)+i_{H *}\left(A^{2 i-2}\right)} \stackrel{\partial_{B}^{2 i-2}}{\rightarrow} \\
& \frac{i_{H *}\left(\operatorname{Ker} \partial_{A}^{2 i-1}\right)}{i_{H *}\left(\partial_{A}^{2 i-2}\left(A^{2 i-2}\right)\right)} \rightarrow \frac{H_{Y-U}^{2 i-1}(\bar{Y})}{\partial\left(H^{2 i-2}(\bar{U})\right)} \rightarrow 0 .
\end{aligned}
$$

Let $C_{4}$ be the complex

$$
0 \rightarrow \frac{H^{2 i-2}(\bar{Y})}{H_{Y-U}^{2 i-2}(\bar{Y})} \rightarrow H^{2 i-2}(\bar{U}) \rightarrow H_{Y-U}^{2 i-1}(\bar{Y}) \rightarrow \frac{H_{Y-U}^{2 i-1}(\bar{Y})}{\partial\left(H^{2 i-2}(\bar{U})\right)} \rightarrow 0 .
$$

There are natural maps of complexes

$$
\chi_{2 i-2}(Y) \leftarrow C_{2} \rightarrow C_{3} \rightarrow C_{4} .
$$

So there are natural maps between the pull-backs of these complexes by the splittings given by $c l^{1}(z)$. Since $C_{2}$ is exact this completes the proof.

\section{Acknowledgements}

Theorem 3.1 used to be only about zero cycles. The author is grateful to Spencer Bloch for pointing out the possibility of generalizing it to cycles of other dimensions. Besides the proof of Proposition 2.1 the author is grateful to Uwe Jannsen for his comments and suggestions on earlier versions of the paper. He also thanks Masanori Asakura for his comments on an earlier version.

\section{References}

[1] A. Beilinson, On the derived category of perverse sheaves, Lect. Not. in Math. 1289 (1987) 27-41.

[2] U. Jannsen, Continuous étale cohomology, Math. Ann. 280 (1988) 207-245.

[3] — Equivalence relations on algebraic cycles, The Arithmetic and Geometry of Algebraic Cycles (Banff, Alberta, 1998), Nato Sci.Ser.C Math.Phys.Sci. 548 (2000) 225-260.

[4] - Letter from U. Jannsen to B. Gross on higher Abel-Jacobi maps, The Arithmetic and Geometry of Algebraic Cycles (Banff, Alberta, 1998), Nato Sci.Ser.C Math.Phys.Sci. 548 (2000) $261-275$.

[5] M. Levine, Mixed Motives, Handbook of K-theory 1 (2005) 429-521.

[6] M. Nori, Algebraic cycles and Hodge theoretic connectivity, Invent. Math. 111 (1993) 349-373.

[7] - Constructible sheaves, Algebra, arithmetic and geometry, Part I, II (Mumbai, 2000), Tata Inst. Fund.Res. Stud. math. 16 (2002) 471-491. 
[8] W. Raskind, Higher $\ell$-adic Abel-Jacobi mappings and filtrations on Chow groups, Duke Math. J. 78 (1995) 33-57.

[9] G. Welters, The Brauer group and the second Abel-Jacobi map for O-cycles on algebraic varieties, Duke Math. J, 117 (2003) 447-487.

Institute of Mathematics, University of Tsukuba, Tsukuba, Ibaraki, 305-8571 Japan

E-mail address: email: kimurak@math.tsukuba.ac.jp 